\section{A0012 Pleth Variability Index versus Stroke Volume Variation as Predictors of Fluid Responsiveness in Prone Position \\ Bhagya R. Jena, ${ }^{1}$ Girija P. Rath, ${ }^{1}$ Vikas Chouhan, ${ }^{1}$ Nidhi Gupta, ${ }^{2}$ Siddharth Chavali, ${ }^{1}$ Arvind Chaturvedi' \\ 'Department of Neuroanaesthesiology and Critical Care, All India Institute of Medical Sciences, New Delhi, India \\ IIndraprastha Apollo Hospital, New Delhi, India}

Background: Prone position is commonly used for spine surgery. This study was designed to investigate the effects of prone position on the accuracy of pleth variability index (PVI) to predict fluid responsiveness and to correlate the changes in stroke volume variation (SVV) with PVI in prone position.

Materials and Methods: After Institutional Ethics Committee approval, 51 adults of either gender, aged 18-65 years, ASA status I-II, undergoing elective spine surgery in prone position were included. Heart rate, blood pressure, cardiac output, stroke volume index (SVI), SVV, CI, perfusion index (PI), and PVI were recorded before and after volume expansion with $500 \mathrm{~mL}$ of hetastarch $6 \%$ given twice; in supine and after prone position. A Masimo pulse oximeter probe with a Radical-7 monitor and a Vigileo monitor with an interface FloTrac transducer were used where appropriate. The above parameters were recorded at different time intervals.

Results: Mean age was 41 years; most were male patients (M33:F18). After second volume expansion, SVI was increased by $9.2 \%$, SVV was reduced by $2.3 \%$ ( $p=0.00$ ), and PVI was decreased by $38.1 \%(p=0.00)$, compared with the previous values. In supine position, there was no significant difference between the area under ROC curve for SVV (0.745) and PVI (0.611) with respect to identifying a change in SVI greater than $15 \%$. The best threshold values to predict fluid responsiveness in supine position were more than $12 \%$ for SVV and PVI. Similarly, in prone position, there was no significant difference between the area under the ROC curve for SVV (0.638) and PVI (0.532). The best threshold values to predict fluid responsiveness were more than $9 \%$ for SVV and $11 \%$ for PVI.

Conclusions: Both SVV and PVI are useful indicators of fluid responsiveness in mechanically ventilated patients undergoing spine surgery. SVV and PVI correlated well, in prone position, with regard to fluid responsiveness.

\section{A0013 Patient Satisfaction Survey on Quality of Anesthesia in Neurosurgical Patients: A Tertiary Care Neuro Center Experience \\ $\underline{\text { Rajesha Srinivasaiah, }},{ }^{1}$ Martin J. Bamber, ${ }^{1}$ D. Vadivel, ${ }^{1}$ Sandeep P. Lakhani ${ }^{1}$ \\ ${ }^{1}$ The Walton Centre NHS Foundation Trust, Liverpool, United Kingdom}

Background: An important aspect of the quality of anesthesia is the satisfaction of patients with their care. Although the patient satisfaction is an important measure of the care they received, it is not an accurate measure of overall quality, safety, effectiveness, or value of anesthetic care. It is also a challenge in itself to measure the patient satisfaction in neurosurgical patients.
Materials and Methods: After obtaining permission from our hospital's clinical governance committee, we conducted a patient satisfaction survey on quality of anesthesia. The survey was conducted for 3 months duration and included 100 randomly selected patients. The questionnaire was given on postoperative day 1 by an anesthetist not involved in their anesthetic care. We used a validated questionnaire developed by Hocking et al, it consisted of 14 questions, and we used the Likert scale to measure the quality of anesthesia. The questions were on preoperative, intra operative and postoperative quality indicators and patiens' overall satisfaction.

Results: All patients were seen by an anesthetist and majority of the patients said they had enough time to ask all questions; however, only $83 \%$ of the patients remembered the discussion of anesthetic risks. We were excellent in our technical ability, friendliness, and addressing the concerns of the patients. $85 \%$ of our patients said they had mild to moderate pain; $97 \%$ of the patients said they would recommend their anesthetist to friends and family. And the overall patient satisfaction score was $99 \%$.

Conclusions: Patient satisfaction survey is an important quality improvement process. Patient's perception of quality of anesthesia depends on the amount of information given during preoperative visit and setting expectations. More importantly, it identifies the strengths and weaknesses of the anesthesia department, and we can learn and improve from our shortcomings.

A0014 Survival and Functional Outcome in Children with Traumatic Brain Injury Requiring Mechanical Ventilation Priyanka T. Nagaraj, ${ }^{1}$ Nidhi Bhatia, ${ }^{1}$ Kajal Jain, ${ }^{1}$ Sunil K. Gupta, ${ }^{2}$ Tanvir Samra ${ }^{1}$

'Department of Anaesthesia and Intensive Care, Postgraduate Institute of Medical Education and Research, Chandigarh, India ${ }^{2}$ Department of Neurosurgery, Postgraduate Institute of Medical Education and Research, Chandigarh, India

Background: Traumatic brain injury (TBI) is one of the leading causes of morbidity and mortality in children. Despite the high frequency of TBI in young children and their precarious developmental status, the recovery and outcomes following TBI in early childhood is not well understood. We aimed to determine survival and functional outcome in pediatric TBI patients requiring mechanical ventilation. Our primary objective was to determine patient outcome at 3 months following discharge from hospital, with secondary objective being characterization of factors which may affect outcome, to determine patient outcome at discharge from hospital, length of ICU, and hospital stay.

Materials and Methods: We prospectively evaluated 50 children (1-10 years of age), admitted to our ICU with TBI. Probable predictors of outcome were abstracted, including Pediatric Trauma Score, clinical variables, Glasgow coma scale (GCS) score, computed tomography evidence of brain injury, and hospital course. Patient outcome was determined using modified Rankin scale at the time of discharge from 